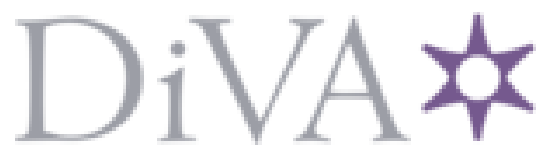

http://www.diva-portal.org

Postprint

This is the accepted version of a paper presented at DIS.

Citation for the original published paper:

Ciolfi Felice, M., Fdili Alaoui, S., Mackay, W E. (2021)

Studying Choreographic Collaboration in the Wild

In: Designing Interactive Systems Conference 2021 (DIS '21) New York, USA https://doi.org/10.1145/3461778.3462063

N.B. When citing this work, cite the original published paper.

Permanent link to this version:

http://urn.kb.se/resolve?urn=urn:nbn:se:kth:diva-295771 


\section{Studying Choreographic Collaboration in the Wild}

\author{
Marianela Ciolfi Felice \\ ciolfi@kth.se \\ KTH Royal Institute of Technology \\ Stockholm, Sweden
}

\author{
Sarah Fdili Alaoui \\ saralaoui@lri.fr \\ Université Paris-Saclay, CNRS, Inria, \\ LISN \\ Orsay, France
}

\author{
Wendy E. Mackay \\ mackay@lri.fr \\ Université Paris-Saclay, CNRS, Inria, \\ LISN \\ Orsay, France
}

\begin{abstract}
Dance making is often a highly idiosyncratic, collaborative endeavour between a choreographer and a group of dancers that constitutes a rich context for designers of creativity-support tools (CSTs). However, long-term, ecologically valid studies of collaboration in dance making are rare, especially when mediated by digital tools. We present a 5-month field study in the frame of a dance course, where a choreographer and six students used a CST originally designed for choreographic writing. We contrast our findings with our initial assumptions about the role of the tool to mediate a diversity of notating styles and hierarchical roles. We highlight the value of and the challenges behind this in-the-wild study in uncovering needs and roles as they emerged over time.
\end{abstract}

\section{CCS CONCEPTS}

-Human-centered computing $\rightarrow$ Field studies; • Applied computing $\rightarrow$ Performing arts.

\section{KEYWORDS}

choreography, dance, collaboration, longitudinal study, creativity

\section{ACM Reference Format:}

Marianela Ciolfi Felice, Sarah Fdili Alaoui, and Wendy E. Mackay. 2021. Studying Choreographic Collaboration in the Wild. In Designing Interactive Systems Conference 2021 (DIS '21), fune 28-fuly 2, 2021, Virtual Event, USA. ACM, New York, NY, USA, 13 pages. https://doi.org/10.1145/3461778.3462063

\section{INTRODUCTION}

Dance making constitutes a rich, complex context for the design of interactive systems and in particular of creativity-support tools (CSTs) for multiple reasons. First, dance making is an art form that is rarely carried out in isolation and often requires time in a dance studio where one or more choreographers interact with one or more dancers. Dancers can fluidly take a variety of roles, acting as, for example, improvisers, interpreters, full co-authors, or all three [33]. They can contribute with conceptual ideas, emotional qualities, concrete body movements, or by solving choreographic problems. Second, the composition of a dance piece does not follow any standardised, universal method and is indeed a highly idiosyncratic endeavour [18, 22]. Each group of artists define their own creative practices, including how (and if) to capture traces of the process, which may involve representing the movement material and documenting the decisions and context that generated

DIS '21, fune 28-fuly 2, 2021, Virtual Event, USA

(C) 2021 Association for Computing Machinery.

This is the author's version of the work. It is posted here for your personal use Not for redistribution. The definitive Version of Record was published in Designing Interactive Systems Conference 2021 (DIS '21), Fune 28-fuly 2, 2021, Virtual Event, USA, https://doi.org/10.1145/3461778.3462063. it. This, in turn, implies finding common ground for collaboration, which typically happens on the base of each dance piece. Third, and interestingly, choreography is a case in which - unlike other creative fields such as contemporary music composition-dance artists do not really need a digital tool to carry on their creative process. However, they are increasingly interested in demystifying their own creative process, examine it, augment it, or even disrupt it with technology [4, 21, 23].

Despite the increasing interest of the HCI community in designing CSTs that accommodate the complexities of dance making, there is still a gap in the CST literature regarding studies of collaboration. According to Dalsgaard [20] "few digital tools [for creative work] are developed with collaboration in mind". And, although a variety of CSTs were designed for dance making, only a few address collaboration from the beginning of the creative process $[16,19]$. Long-term deployments of technology specially designed for dance making in ecologically valid studies could illuminate different ways in which collaboration unfolds among dance artists. However, such studies also remain rare [13, 38].

This paper focuses on a 5-month field study with a choreographer and six pre-professional dance students who used Knotation [19], a CST for choreographic writing, in the frame of a course on dance and technology in a conservatory in Paris, France. We had designed Knotation for and with choreographers $[18,19]$ but the prototype had not been deployed as a fully-fledged tool in real-world settings without artificial tasks and within an ecosystem of other analog and digital tools. Before presenting our analysis, we disclose our initial assumptions as researchers studying collaboration in dance making. We then offer our findings, which nuanced or contradicted our assumptions as the deployed technology played a wider variety of roles than expected but did not attenuate existing power dynamics or style differences among collaborators. We discuss the value of this type of field study, the challenges that it posed from the researchers' perspective, and how our results regarding what the technology did not mediate is not a problem to solve, and instead a complexity to take into account when designing CSTs for dance making.

\subsection{Authors' positionality}

The second author of this paper is an HCI researcher, choreographer and dance educator. The longitudinal study that we present was carried out was during a course that she gave on Dance and Technology at a dance conservatory in Paris. The goal of the course was to collectively create a choreographic dance piece along with the students, involving digital technologies in the making and in the performance. The final piece was performed by the students at the conservatory's end-of-the-year show. While we, authors of this paper wrote it from a research perspective and as academics 
doing field work, we recognise and carefully include the experiences of the second author as both a researcher and a creative user of the technology in the artistic context that we investigated. Our intention was to study a real dance context, using both a first- and a third-person perspective, and reflecting on participants' and on our own experiences of how technology intervenes in crafting and documenting the artwork. We do not consider the second author's involvement as a bias because this concept is not relevant to this paper, for two reasons. First, we do not intend to present our findings as an evaluation of Knotation. Second, any field study is necessarily influenced by the researchers' perspectives and even by their presence in the setting, as well as by the participants' expectations and sense of duty [10]. Instead, our work leverages on the connoisseurship and involvement of the second author, both as an expert user of the technology, and as a researcher reflecting on the experiences that it has provoked. Thus, we offer this as a concrete case study about a choreographic creative process when mediated by a digital tool in a real collaborative setting.

\section{RELATED WORK}

\subsection{Creativity-support tools for dance making}

One of the reasons why choreography is of particular interest for $\mathrm{HCI}$ is that it presents additional challenges with respect to other creative practices, because of the extreme diversity across choreographic approaches and because dance is based on human movement, which is complex, multimodal, and hard to capture [28] Multiple studies, design approaches and technological systems were born from the interest in HCI to support dance making. This previous work has looked at ways to support dance creation [12, 48], improvisation [40], analysis [27] or annotation [25], among others (see [2] for an extensive review). At the same time, deLahunta and Shaw [23] recognised a shift towards multidisciplinary collaboration between dance artists, dance researchers, and makers of interactive technologies, stemming from two sources. One source was the change in what constitutes a useful resource for researchers working in multidisciplinary areas. The authors noted that the creative process in artistic fields, rather than the creative product itself, started gaining attention as a source of knowledge for other fields $[1,39,40]$. The other source was the artists themselves wanting to share and reflect on their creative process. deLahunta and Shaw speculated that this could be a response to researchers' increasing interest, or an attempt to spark innovation.

In HCI in particular, these multidisciplinary collaborations result mainly from the design of CSTs for choreography. CSTs can be defined as "any tool that can be used by people in the open-ended creation of new artefacts" [15]. Within this trend of research, we find relevant the CSTs that address the collaborative aspects of choreography, i.e. those that are designed for or acknowledge the existence of collaborators and their roles, targeting, e.g., dancers $[44,56]$, choreographers [43], or both [16, 19, 50]. Long-term studies with CSTs tools are lacking in general, and particularly those run in the wild. A remarkable exception is Carroll et al.'s [13] study of three full dance productions using the Choreographer's Notebook [50], which we expand upon in the next section.

\subsection{In-the-wild research in $\mathrm{HCI}$ and dance}

In the last decades, HCI research has highlighted the importance of in-the-wild exploratory studies. Rogers [46] defined research in the wild as involving designing, prototyping, implementing and studying technology in situ, outside of the laboratory, and with the goal of articulating how it affects people's everyday experiences and appropriation practices. More specific to the performance context, Benford et al. defined performance-led research in the wild as the set of explorations "concerned with collaborating with artists to create cultural experiences that are deployed "for real", typically in a bona fide cultural venue such as a gallery, theaters, theme park or festival, to be experienced by the public who should see them as cultural artifacts rather than laboratory prototypes." [3, p.6]. This approach has been followed by several researchers who used performance as an experimental ground to investigate viewer's experience [53], audience engagement [24], participation in performances [14], interaction between sonic artefacts and collaborators in a dance production [42], idiographic design strategies for performance-oriented technology [32] and to exhibit the tensions in using technologies as part of dance stages [26].

As much as this literature shows the value of research on performance to be held in the wild, it is mostly concerned with technologies as creative artefacts on stage. In fact, very few studies on performance or dance in the wild include technologies meant to support the creative process throughout. The most relevant are the series of deployments of the Choreographer's notebook (ChoNo) [50], a web-based system that allows dance artists to analyse and annotate rehearsal videos. Carroll et al. [13] examined the sociotechnical effects of introducing this technology in three full dance productions. Although designed for use outside of a dance studio, they reported how the choreographer collaborated with the dancers by projecting the content of their ChoNo during rehearsals and going through the annotated videos with them. Their findings also highlighted the inherent hierarchical nature of the collaboration in dance, as they showed how choreographers made most of the comments on dancers' videos, while dancers did not correct each other. More recently, Rivière et al. deployed MoveOn [45] - a system that allows users to decompose and annotate dance videos-during a year-long field study of a dance company re-staging a piece from the contemporary repertoire. The authors showed that participants created and appropriated an ensemble of heterogeneous complementary artefacts in addition to the introduced system. They also showed how their results unfolded in time, allowing for a deeper articulation of the interactions between users and technologies in the context of collective practices - which further highlights the importance of longitudinal studies when deploying CSTs in dance making.

\subsection{Diversity of roles and relationships in dance}

Numerous researchers have explored the complexity of dance making, particularly the collaboration and shifting roles of dancers and choreographers as they work together. For example, Blom and Chaplin [5] noted that Doris Humphrey was one of the first choreographers to consider the dancers' contributions, including their 

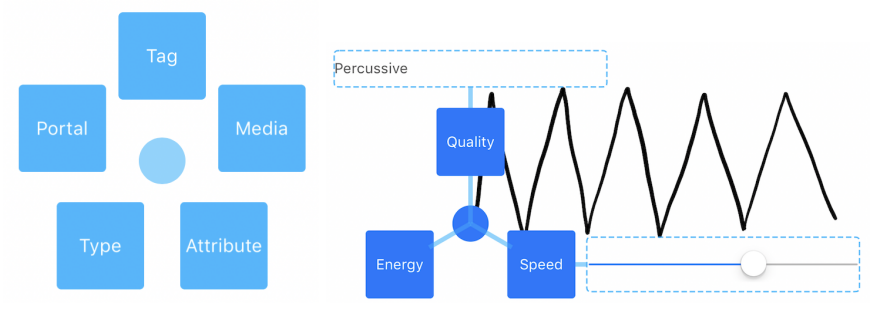

Figure 1: Knotation. Tapping a newly-created knot reveals the menu of available features (left). Knot attached to a zigzag trajectory: Tapping the knot reveals three attributes. Here, the knot defines a "percussive" quality, with a slider to indicate speed (right).

technical skills, personality, style, and creativity. Rowell [47] analysed the status of contemporary dance in Europe and the UK, noting an emerging "new way of looking at the dancer (...) whose agency within the dance statement is finally acknowledged". Rowell argued that, implicit within this view, is a new status for dance as a collaborative art. Similarly, Klien [36] stated that in this new political context "dancers are no longer 'employed to perform"” and have to negotiate "their personal freedom and subjective reality within a larger group". Also in the context of the UK's contemporary choreographic scene, Butterworth [11] identified five "processes of collaboration" between choreographers and dancers, and proposed a framework called Didactic-Democratic Spectrum with different degrees of collaboration between dance artists. Later on, Gibbons [30] described the choreographer as a "curator" who "selects, assembles, and sequences movement material, structures, and conceptual ideas". Gibbons talked about choreographers and dancers generating material together and inspiring each other, and noted that the responsibility emerges in the "editing process", as guiding principles and questions evolve. From a cognitive science perspective, Kirsh et al. [35] conducted an ethnographic study with the world-famous choreographer Wayne McGregor and the dancers from his company. They collected video, field notes, interviews, motion capture data, psychological tests, diaries, and notebooks. As a result, the authors captured the methods used by the choreographer for creating dance with dancers: showing, making-on, and tasking. Each method presented different levels of agency and dancer creativity, and involved multiple communication modalities, such as the vocalisation of dance movements and rhythms.

In HCI, Hsueh et al.'s study [33] showed that choreographers and dancers shifted roles fluidly throughout the creative process. The creator's roles ranged from author, curator, planner, to researcher, while the performer's role shifted between interpreter, creator, improviser and informant. They argued that existing CSTs tend to reinforce singular roles and linear tasks, whereas the fluidity and "slippages" observed in real-world creativity should be accounted for in design, as they open up creative opportunities. Indeed, while the field of dance has acknowledged the blurry boundaries between roles in dance making, most technologies for dance continue targeting the choreographer as the maker and sole inventor of movement.

In summary, there is a growing interest in HCI to support dance making, and in the dance field to examine the creative process, and to partner with researchers. However, existing CSTs either do not address the collaborative nature of choreography, or do so in rather advanced stages of the creative process, when collaboration patterns have already emerged. On top of this, most CSTs for dance making have been deployed in one-shot experiments or semi-structured studies, with few longitudinal studies in the wild. To help fill this gap, we present a deployment of Knotation [19] in a five-month study with a choreographer and six dancers.

\section{KNOTATION}

Knotation [19] is a pen-based mobile tool that we designed with and for dance artists (choreographers and dancers) in the frame of a long-term participatory design process [17-19]. In conversations with dance artists, and addressing a gap in the CSTs literature, we as designers defined the goals of building an open-ended, advanced yet light-weight software tool that could support exploration and documentation of choreographic ideas from the beginning of the creative process. More specifically, we wanted to design a tool that made knowledge about the dance piece available to the users, allow for multiple representations of choreographic ideas, and facilitate transition between abstraction and detail.

Knotation runs on Apple's iPad Pro, which can be brought into the dance studio. Choreographers can sketch with the Apple pencil, as well as link and interact with their own choreographic annotations without enforcing a particular dance representation. Users can insert pictures and videos, captured in the moment or imported as recorded material. Knotation keeps a version history for each document, and to facilitate collaboration across iPads, it can import content from another Knotation's document into the current canvas.

Ciolfi Felice [17] related Knotation's design to Shneiderman's guidelines for CSTs [49], arguing that the tool: supports exploratory search, since users can manipulate personal representations of their ideas directly; facilitates the generation of alternatives, since users can lay out such ideas in different configurations that can be visualised simultaneously; and provides users with a rich history, as they can revert to previous states of the canvas. The most relevant for this paper is Ciolfi Felice's reflection about Knotation enabling collaboration, as "it is a mobile tool that can be physically shared among collaborators, it does not impose a formal notation language requiring previous training, and it allows quick capture of in-themoment ideas and dance sequences with dancers in the studio" [17, p. 158]. We consider this last reflection as an assumption of the designer, given that Knotation had been used by choreographers in 


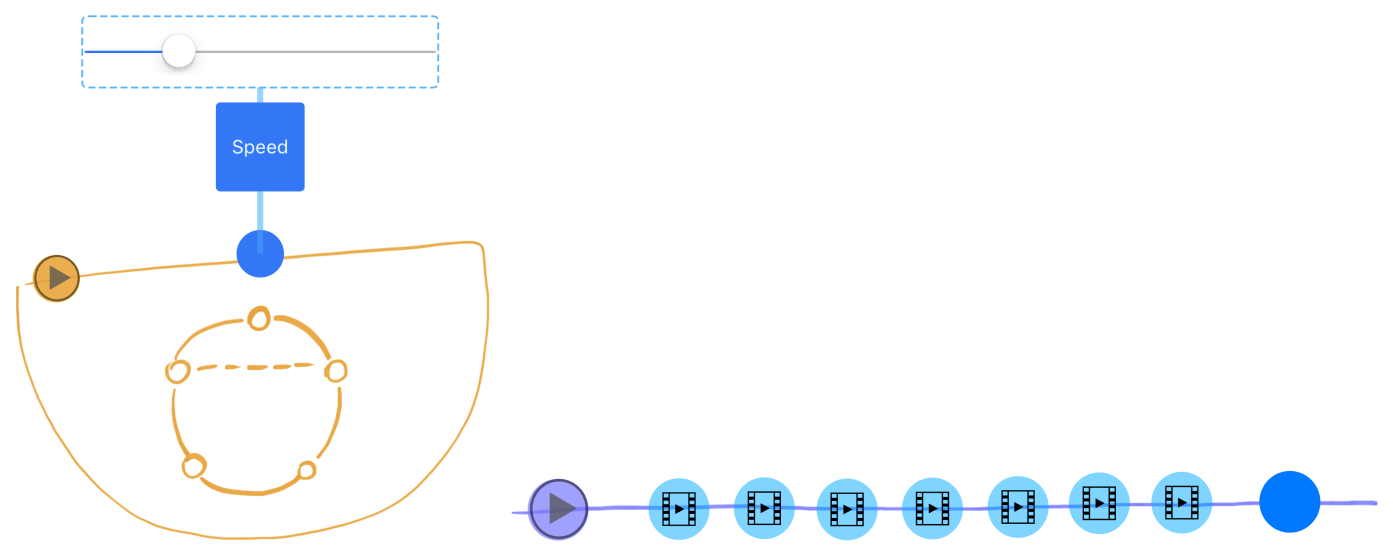

Figure 2: Knotation. A floorplan with a speed knot controlled by a slider (left). A timeline with video knots and a speed knot (right).

semi-structured observations [19], but its use in real-world settings had not yet been explored.

\subsection{Features}

The following features are relevant for the field study; the full set of features is described in [19]. In Knotation, the user creates and interacts with objects that can be moved, cloned, edited, and deleted.

3.1.1 Sketches and knots. When the user sketches with the pen, Knotation groups the strokes into choreographic objects (representations of choreographic ideas at any level of abstraction). The user can add functionality to their sketches through long-pressing, producing a dot called knot. Knots can also be created in the flow of writing or sketching by simply dwelling on the surface. The user can then tap a knot and select a function from a menu (Fig. 1 left). Just like sketches, knots are also objects. They can contain multiple attributes, whose values can be set via a controller (Fig. 1 right).

3.1.2 Multimedia content. The user can incorporate multimedia content by associating a knot to an image or video in the iPad's library, and interact with them through direct manipulation. For example, a user might want to sketch a floorplan (a diagram that represents dancers' spatial trajectories, as seen from above) and attach a rehearsal video of the corresponding dance fragment, to keep them linked: They can add a knot to the border of the floorplan, and import the video into the knot. Then they can play the video by tapping on the knot.

3.1.3 Floorplans and timelines. Knotation provides support for floorplans. Creating an interactive floorplan begins by drawing a closed (or almost closed) area, and attaching a floorplan knot. The border turns orange, indicating that the figure is now interpreted as an enclosed 2D space. Any strokes within this figure are considered trajectories, and are also rendered in orange. Tapping on the floorplan knot animates each trajectory in the direction in which it was drawn. Users can modify the speed of the trajectories by attaching a speed knot to the floorplan's border or to a specific trajectory (Fig. 2 left). Alternatively, users can apply a duration knot to specify the duration of one or all trajectories. When doing so, Knotation calculates the speed of each trajectory in the floorplan such that they all finish at the same time.

Knotation introduces interactive timelines, which let choreographers define temporal sequences. Creating a timeline consists of drawing a stroke of any shape and attaching a timeline knot, which turns the stroke violet. Users can then add any type of knot to the timeline (Fig. 2 right). Users can also create a new timeline by drawing a stroke across an existing set of knots and attaching a timeline knot to the stroke. Tapping on the timeline knot displays the video knots in the order specified by the direction in which the timeline was drawn. The timeline plays the videos either at normal speed or at a speed determined by a speed knot. Users can reorder, edit, clone, attach, detach or delete knots, even as the timeline plays. A single video knot can be attached to multiple timelines, which, for example, lets users explore different combinations of fragments in different orders.

\section{FIELD STUDY WITH A CHOREOGRAPHER AND DANCERS}

The field study was set up to follow a course on Dance and Technolog $y$ at a dance conservatory in Paris. The course was given by the second author between October 2017 and March 2018, was optional for the post-graduate conservatory students, and did not involve grades. The course design had two parts: Part 1 consisted of a 3-hour class per month over five months; Part 2 spanned five days, four hours per day. Students could enrol in only the first part, or both. During Part 1, dancers learned theoretical aspects of choreography, such as Laban efforts and qualities [54], and put them into practice by working in groups to compose choreographic fragments. During Part 2, dancers collectively created a contemporary choreographic piece to perform at the conservatory's end-of-the-year show. The final piece included diverse technologies brought by the teacher such as interactive visuals, vibration sensors, and live electronic music. Fourteen dancers enrolled in Part 1. Six dancers also enrolled in Part 2, so we focus on them in our data analysis. 


\subsection{Participants}

The second author of this paper took part in the study as a participant, since she was the teacher in charge of the course. She had 8 years of experience as a professional choreographer. A total of six dancers (five women, one man; ages: 19-32) followed both parts of the course and performed in the final show. They had between 5 and 16 years (median: 14.5) of dance practice, including their studies. Two wanted to become professional choreographers in the future, and two planned to be professional dancers. Four had some experience with dance making, but not in professional contexts (one for ten years, and the rest for three years or less), and two of these had also taught dance before ( 4 and 8 years).

\subsection{Study design}

We designed the study to avoid increasing dancers' workload. The length of participants' interactions with the first author during the course consisted of debriefings and a one-time interview, were kept to the minimum, and within the hours in which dancers were normally at the conservatory. Naturally, participating in the study was not required for taking the course. The authorities of the conservatory were enthusiastic about us running the study, and invited us to demonstrate the tool and the generated scores at the endof-the-year's show. The study was part of a larger IRB-approved project studying creative practice (ERC no. 321135, CREATIV). Still, we defined a specific study protocol in concordance with it, and obtained written informed consent from each student. The form specified that participation was voluntary and with no additional risks, and that the data would be anonymised unless they requested to be named (a common practice in art-related research) and kept safe.

4.2.1 Before the start of the course. The teacher organised a first meeting with the dancers at the conservatory, where she described the learning objectives and the available technologies. The first author attended this meeting to introduce the goals of the study, and give a demonstration of Knotation. Dancers had time until the next session to decide whether they wanted to participate in the study.

4.2.2 Part 1. Once the course started, each class was held in the dance studio assigned to the course. The first class of Part 1 (which we abbreviate P1C1), dancers read and signed the informed consent. The teacher asked them to divide in groups of three or four people. The first author gave one $\mathrm{iPad}^{1}$ and one Apple pencil to the teacher, and to each group, and trained them in the use of Knotation. Then, the first author would observe each class without actively intervening, taking notes and very sporadically taking pictures. Dancers were told that while in the studio, they could use the iPads at any moment. However, they did not have access to the devices in between classes. Other interactive technologies were present in the setting, since the course was about dance and technology. We focus on Knotation in our analysis while recognising that it sat within an ecosystem of analog and digital technologies. To minimise the chances that the dancers felt observed, the first author was the only non-dancer present in the studio. Towards the end of each class, the teacher would debrief the session, and the first author would

\footnotetext{
${ }^{1}$ The iPads were 12.9 ", ran iOS 11 , and had Knotation installed.
}

ask each group to explain what they had created in Knotation, if anything.

4.2.3 Part 2. During the intensive week, as instructed by the teacher, dancers stopped working in groups and divided into pairs. Each pair was to compose their own choreographic fragment (a duo). Then they integrated these fragments into the global structure of the piece created by the teacher, and learnt some sequences that they would all perform together (also created by the teacher). In the middle of the week (P2C3), the first author interviewed each dancer for approximately 10 minutes, using a variation of the critical incident technique [41]. She asked them to talk about recent, memorable stories of collaboration with other dancers and with the teacher, as well as stories related to Knotation and any of the available technologies.

\subsection{Data collection and analysis}

At the beginning of each class, the first author placed a camera on a corner of the studio to record video and audio, and took notes. She limited the number of pictures and close-up videos to avoid distracting the participants, and filmed the interviews avoiding their faces. Since participants spoke French, she anonymised and translated the raw data to English.

We based the analysis on interview transcripts and the first author's field notes. We first used an inductive approach to code the full dataset, inspired by Braun and Clarke's thematic analysis [7, 8]. We were open to any type of patterns and surprises in the data, and were not looking through the lenses of our initial assumptions. Still, our positionality as authors, artists and educators (Section 1.1) and the Western cultural context of the research affected our interpretation of the data and what we considered interesting. We decided to focus our analysis on stories of collaboration between participants, with and without technology, seeking to capture details on practices, roles, and needs, as well as breakdowns and bright points. While we inductively coded the dataset, we did not construct a set of inductive themes as our main outcome, because we found it more suitable for our case to structure the findings as responses to our three initial assumptions, which could be considered a deductive approach [7]. We also added post-study reflections - which were not part of the original dataset but further contextualise our findings - from both the first and the second authors. The goals of these reflections is to explicitly state our first- and third-person experiences and perspectives of the study. We carefully indicated in the text if the quotes were provided post-study and by whom.

\section{INITIAL ASSUMPTIONS}

We first present our initial assumptions about how the tool would mediate collaboration in a real-world setting, in particular, its role as an exploration and documentation support, its potential for democratising the creative process, and its influence in setting a common annotation approach. We then contrast these assumptions with what we were able to observe in the wild, which uncovered new needs, roles, and relationships between participants and technology, and between each other, that emerged and changed over time. 


\subsection{Assumption 1. Mediating exploration and documentation.}

We expected that dropping Knotation into a collaborative setting with a choreographer and a group of dancers would result in the tool actively mediating both the exploration and documentation of early-stage creative ideas. For example, we imagined dancers exploring different movement ideas with their bodies and with the tool, in a back-and-forth process. This assumption stems from the original motivation behind the tool and our design iterations in the frame of an intensive, long-term participatory design approach with contemporary dance artists, most of whom were both choreographers and dancers [17-19].

\subsection{Assumption 2. Democratising the creative process.}

We were aware that deploying Knotation in the context of a dance course implied inserting the technology into an inherently hierarchical structure, where the choreographer played the role of teacher, and the dancers were students. We expected this distribution of power to affect collaboration to some extent. Carroll et al.'s field study with the Choreographer's Notebook [13] had found that the use of the technology solidified the hierarchical differences between the choreographer and the dancers, given that it was the choreographer who was annotating dancers' videos with corrections after the dance sequences were generated and rehearsed. The dancers, on the other hand, did not comment on each other's videos. We hoped that providing dancers with an interactive technology to support documentation of early-stage ideas, before dance fragments are actually performed and captured on camera, would give dancers more agency in the creative process, especially regarding ownership of movement material. Thus, our assumption was that the CST would capture traces of dancers' explorations, which could lead to a documented contribution from them from the early stages of the creative process.

\subsection{Assumption 3. Generating common annotation policies.}

Knowing that notating dance is a highly idiosyncratic activity and based on our previous work with choreographers who defined their own annotation practices and even languages that dancers and other collaborators learnt to read and interpret [18], we expected that having a shared interactive tool to support this process would result in an agreement on a common notation policy, and act as a boundary object [51] for collaborators. For example, we thought that dancers would either appoint a designated notator among members of each group, or collectively decide how to notate their dance fragments and actively enforce this policy, in order to be able to revise previous material along the 5-month course, and/or contribute to the creation of a unique, shared digital score.

\section{FINDINGS}

We now present our findings in the wild by contrasting them with the assumptions above.

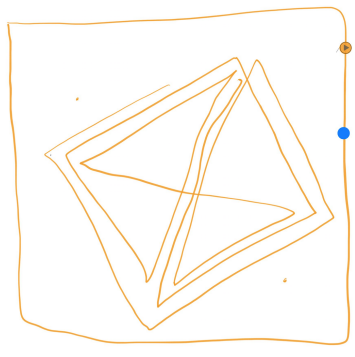

Figure 3: The teacher explored the dynamics of the trajectories on a floorplan representing the "the walk" dance sequence.

\subsection{The technology played a wider variety of roles over time.}

Instead of simply supporting Assumption 1, we found that Knotation mediated different aspects of collaboration as the course unfolded, according to evolving needs and roles of participants. Whereas at the beginning of the creative process it did not mediate collaboration between participants, it later supported horizontal collaboration between dancers, and at the same time, vertical collaboration between them and the teacher.

6.1.1 Technology not mediating collaboration. In Part 1, Knotation was used in ways in which it simply did not mediate collaboration between participants, given that participants themselves were not actively collaborating across groups nor with the teacher. The teacher used the technology to explore creative ideas, while the dancers used it to document final choices, as well as to individually learn or revise previously created material.

The teacher used Knotation in an exploratory way, to spark choreographic ideas. For example, she showed the first author a floorplan for a dance sequence she called "the walk" (Fig. 3): "This is where Knotation is interesting, because I was able to do this, and I think I couldn't have done it (...) if I was to do it on paper... Because I was able to play it and I saw: Oh, this is creating a nice pattern! And I was able to try it with the dancers". However, she also mentioned the lack of time to try different alternatives in Knotation: "If I were in my own studio and I had two weeks of rehearsals I could do multiple of these and try them out. (...) But here I have only four hours per afternoon for a week, so I'm like: Here, these structures, take them and then go to the next thing". This result is in line with Singh et al.'s observations [50] on studio time being too expensive to spend in systematic exploration of ideas, which could instead be done elsewhere with the help of a CST.

In Part 1, the teacher decided to set a shared dynamics for the work. For example, she would often ask each group to show their fragments to the others, otherwise they tended to work isolated from the rest. Dancers, in this part of the process, used the technology mostly to document their fragments, following direct instructions from the teacher. They worked in a highly embodied way when exploring movement variations: They would try alternatives with the body first and then use Knotation to persist only the final choices. P3 stated: "I think it's a novel way of preserving a document, where we can write down more things than with video". P4 explained 

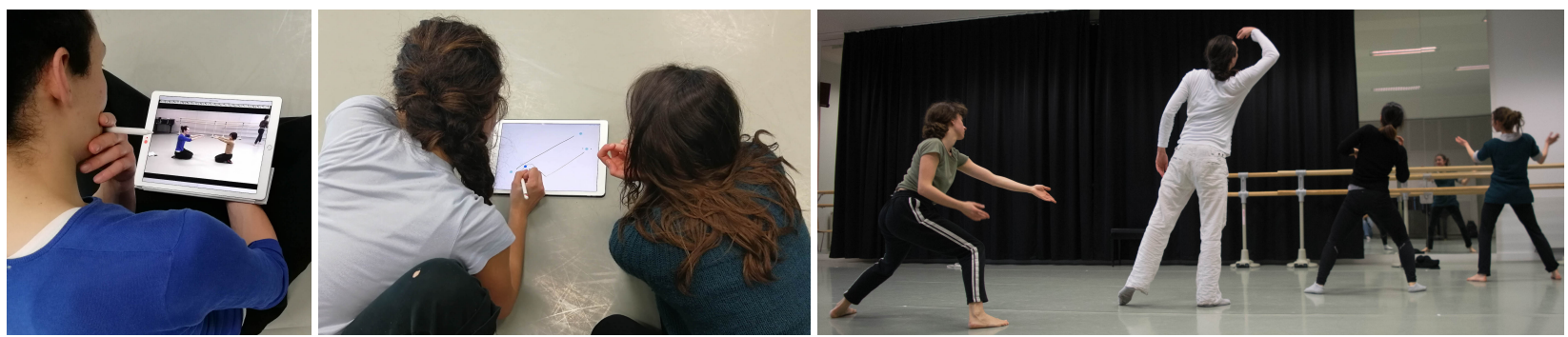

Figure 4: A dancer revises a video of a dance sequence to examine details (left). Dancers draw spatial trajectories in Knotation to document their duo (center). Dancers rehearse a common phrase in Part 2 (right).

why she and P5 did not use interactive features such as floorplans and timelines: "We didn't really have the need... We used the iPad as a digital notebook (...) to remember decisions".

In the interviews, dancers reflected upon documenting their dance fragments. P5 explained: "The fact that we with P4 used the iPad at the end of the session... it was a bit like homework (...) It was OK because it forces you to make a memory effort, so it's reworking the thing in a different way...”. Perceiving the interaction with technology as homework was already reported by Carroll et al. [13] in the context of dancers using the Choreographer's Notebook. Moreover, Latulipe [37] reflected on the trade-off implied in the use of the ChoNo: While it provided dance artists with rich exploration and communication of their work, it also had a cost for users in terms of their work-life balance. In contrast with the ChoNo case, P5 added: "We would need a notator, someone who does that job while you're creating... It's hard to introduce the object [the iPad] during the creation process...".

Not seeing documentation as a need did not surprise the teacher, taking into account that this was, for some of the dancers, the first time that they had to produce a score for a relatively big piece. As they had not experienced the need of revisiting the documentation of a past project, they did not have the same expectations as the teacher about keeping representations of their movement ideas, let alone interactive representations. The teacher reflected about the importance of teaching students the value of documentation in its political dimension: "It is key to make the dance exist beyond the actual movement. Leaving a trace in history, persisting it, being able to transmit it and to transform it into knowledge. The political power of documentation lies exactly in that the document materialises the knowledge emerging from making - a knowledge that is less visible in the sole experience of moving. To put it simply, the document is a traceable representation of the knowledge that is generated by the body, that is otherwise ephemeral and withers with the action of dancing" (post-study reflection).

Dancers also used the technology as one more means to learn dance phrases, sometimes individually and sometimes within their group/pair. For example, they spontaneously used Knotation as a self-reminder at the beginning of each class. Most groups often came back to their iPads on the floor during the class to check a detail or write something down. For example, the first author observed P2 and P3 playing a timeline in Knotation and then performing the movements. At some point, they watched several videos at the same time, to compare movements. Then, they left the iPad and started dancing, trying to remember the movements together and exchanging comments about the details. Similarly, P1 reflected on the value of having the sequences filmed from class to class: "A very happy moment, because it allowed me to progress faster, to unblock the memory also, was having the videos, of course... Because it comes back instantly once we have them". P1 used the technology to carefully revise choreographic material: He recorded movements in slow motion, imported them into Knotation and played the videos to check interesting details (Fig. 4 left). In addition, floorplans helped P1 learning a specific choreographic aspect: "I have a lot problems with space, so the fact of having a diamond on the squared room, it would have been harder for me to understand, (...) So that I think that's really an added value [of Knotation] (...) and the fact that it's interactive and progressive. (...) The fact of being able to see it several times without bothering anyone, replay it, and see the traces in space as someone that advances... The memory of images is one thing, and the memory of visualised movements, it's completely another thing". P1 also referred to the importance of interactivity in this context: "Video, accumulating media, is good, but... What is really good is the interactivity, or reality, well, not augmented, but... things we couldn't do by hand... It's like if the paper was alive... Seeing a pencil that draws like Harry Potter".

6.1.2 Technology mediating horizontal collaboration. Following the course over time allowed us to observe how an initial conflicted relationship to using technology changed considerably throughout the process, as a horizontal collaboration between dancers emerged. The first author observed that dancers' feeling of belonging to the group and to the creative process progressed throughout the course and increased their engagement with the piece - a perception that was confirmed by the participants. In Part 2, dancers incorporated documentation practices into their routines at their own initiative (Fig. 4 center). At the same time, interaction across groups increased considerably, and dancers started showing solidarity with each other, for example, by teaching phrases and helping others master certain movements, mediated by Knotation (Fig. 4 right). Dancers used the technology to transmit the generated material whenever a member of their group annotated something on their own, or when a dancer missed a class. When P1 was reassigned with P2 for the duos, P2 had to learn new material from scratch. P1 talked about the experience of using Knotation to transmit the phrases: "I changed partners several times. (...) The videos allowed me to transmit quickly. First I showed a scheme to P2, then the chain of videos too, then the animated chain, to see how the two people advanced... And 

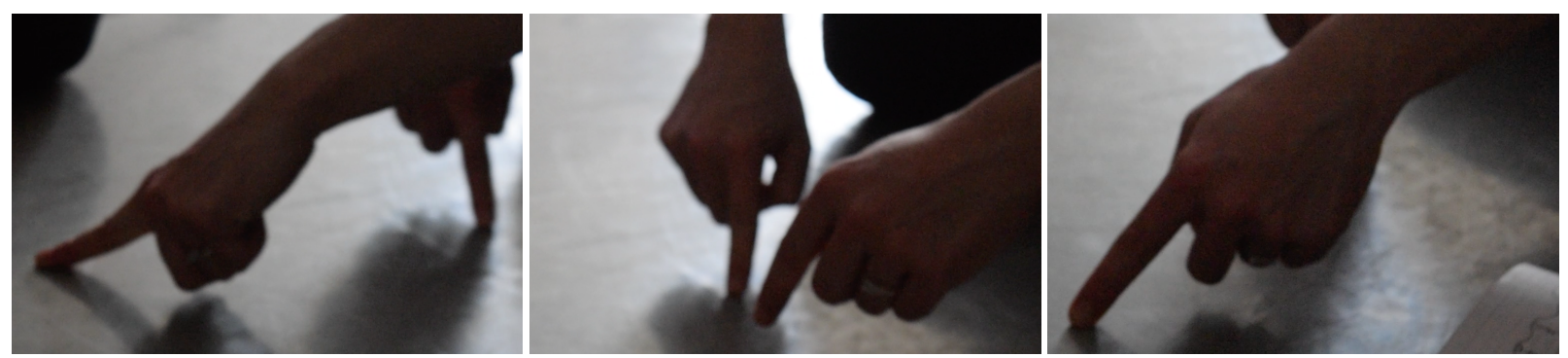

Figure 5: The teacher and the dancers represented and discussed dancers' trajectories by moving their fingers on the floor.

that went very fast. We can consider that Knotation fully fulfilled the job because I used the score for P2 and she didn't know anything about the score, and she understood everything". P2 also referred to this story from her own perspective: "P1 placed the iPad and told me 'I let you to it', and I succeeded at understanding what P1 had done". However, she added: "It helped me to have a first vision of the duo, but it required P1's explanation. If P1 hadn't been there, if I had had only the iPad, I think I wouldn't have succeeded at understanding everything. In fact it's the videos that helped me".

6.1.3 Technology mediating vertical collaboration. Even if dancers became engaged in documenting their creations, the hierarchical roles were always present, and the technology complied with this hierarchy: In Part 2, the teacher proposed centralising the global score on one device and asked the dancers to stop updating their compositions on their iPads. She then used Knotation intensively, creating a "global score" and adding each duo's score into it (Fig. 6). From that moment on (P2C3), her annotations in Knotation implicitly became the one source of truth in terms of score, and constituted a shared object (and place) to which the dancers would spontaneously come and sit around on the floor.

Interestingly, dancers and the teacher also used Knotation to mediate their exploration of ideas together. For example, the first author observed the teacher and two dancers discussing possibilities for a transition: They placed their fingers vertically on the floor to indicate dancers' initial positions, and moved them at different speeds to indicate the trajectories (Fig. 5). The teacher proposed to continue in Knotation, so she created a floorplan illustrating her idea and played it. As they discussed alternatives, she deleted the trajectories. Then the students drew their own trajectories in turn, to communicate their proposals, and played the floorplans. Each time they would suggest a new configuration, they would delete their previous trajectories. At the end, the teacher redrew only the one she chose in order to document it.

The insertion of technology also affected the way in which the teacher transmitted the choreographic material to the dancers, although not from the beginning of the process. In Part 1, the teacher worked exclusively with a paper notebook as the external support for the choreographic material she created. She transmitted it vertically, by showing her annotations (mostly diagrams and text), but also by talking, gesturing, and marking movements. The dancers simply asked questions to check that they understood.

As described before, in Part 2 the teacher used Knotation extensively to create and then vertically transmit the global structure.
One dancer appreciated that the teacher had included floorplans and told her: "Normally the directions on the diagonal would be a nightmare for me. It was really great that you were able to prepare it in advance, that you did it on the iPad. It would have taken me [a long time]. (...) I think most of my problems with directions were solved because of what you did. (...) It's great" (P1). The teacher also conducted feedback sessions around the rehearsal videos that she incorporated to her score each day, indicating corrections and things she liked. This vertical transmission of material mediated by technology had a positive effect in terms of engagement with the creative process. In particular, the teacher noticed a change in the dancers' attitude in $\mathrm{P} 2 \mathrm{C} 3$, after she presented the "global structure of the piece": "For me there was a difference today in terms of their engagement with the piece (...) I guess maybe today they started seeing where it was going... They started to think the piece might look good". For example, $\mathrm{P} 4$ said at the end of that class: "I think we arrived to a moment where the structure is very clear, we know what we have to do, and this allows us to find more links, to find meaning in our actions, in the chaining of things".

After this class, and as dancers' engagement increased, the teacher took progressively fewer notes, relying on the sense of membership that emerged. She said she trusted in their responsibility to remember the decisions made as a group: "In dance you're relying on people and they rely on you". As dancers got attached to their own ideas, their suggestions became negotiations. Starting in this class, participants often resorted to Knotation to mediate such negotiations. They would all sit on the floor around the iPad at least once per class, in order to watch the videos they had shot for each part of the piece. The teacher would play the videos in Knotation, pausing them often to point at the details she wanted to highlight. She would perform mid-air gestures and mark movements to indicate corrections, proposals, and aspects she liked. The dancers would detect eventual mistakes and make their own suggestions. P4 referred to the negotiation with the teacher in this context: "We compromised. She accepted things, we accepted things. We saw what works and what doesn't. (...) The videos helped a lot". This finding shows once again how the technology complied with existing hierarchical roles, while offering support for negotiation, which is an important aspect of collaboration when such power differentials exist. 


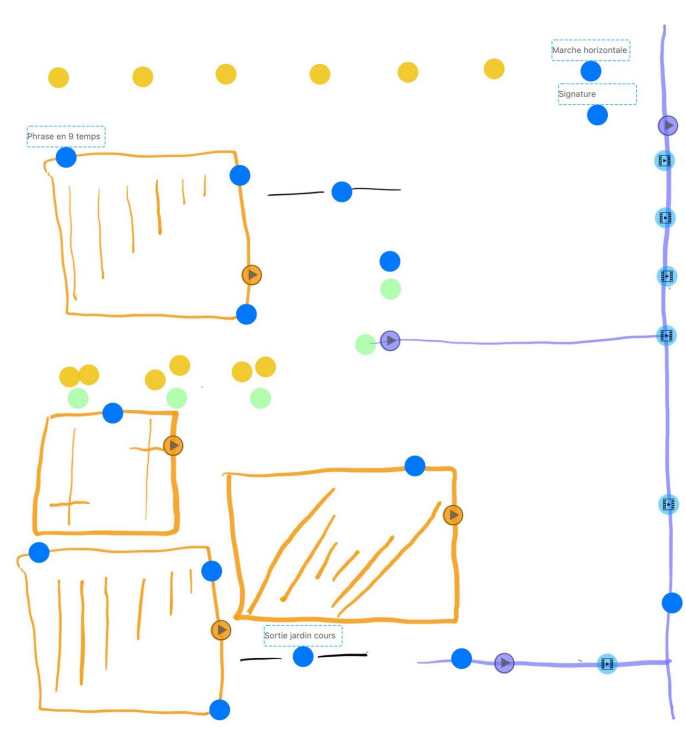

Figure 6: The teacher created a global score for the piece and imported dancers' duos.

\subsection{The technology did not democratise the creative process.}

Our results did not comply with Assumption 2. They showed that hierarchical roles, as well as participants' perceptions of such roles and conceptions of what collaboration entails, directly impacted their use of and relationship to the technology introduced, as reported in Section 6.1. It caught our attention how collaborators had contrasting perceptions of their roles, touching on concepts of agency and trust. In particular, our interviews with dancers captured a perceived a tension between their own agency and the teacher's hierarchical role. For example, $\mathrm{P} 4$ explained: "She [the teacher] gave us the chance to create and at the same time she had a very particular idea in her head. So maybe that was hard to manage because on the one hand she would tell us 'yes, go on, it's yours, it's your material', and then no...”. The teacher also perceived such tension and responded to it: "Because they lost the focus I was like: $\mathrm{Ok}$, I'm taking this in charge. I'm making the whole score (...) In dance, there always should be someone who says: that works, that doesn't. Being in charge also takes some agency from them". Moreover, the teacher reflected on a component of trust that needs to complement the choreographer's hierarchical role for the collaboration to be successful: "There's something about collaboration: Sometimes you have an idea, but in order to get to it, there are so many steps you need to explore, and the dancers don't know that idea you want to get to, but they have to trust you". Interestingly, the teacher made a distinction between generating material and making a score, and reflected about dancers' agency in this context: "I give them agency, they create the material, but I'm always the score writer". She said that even when collaborating with professional choreographers: "Most of the score is mine. It's also something I really like to do. I'm not sure this is a collaborative activity... Generating material, yes, but writing a whole score, no".

\subsection{The technology did not bridge the diversity of notating styles.}

Our results did not comply with Assumptions 3 either. Unsurprisingly, dancers either did not have a personal way of notating dance, or they had an idiosyncratic one that was different from the teacher's. Still, in both parts of the course dancers had to share one iPad within their group. Some groups used the pen in turn, without much planning, while others discussed and decided on a common policy for their annotations. In particular, P2 said that in her group they all had "different writing methods" (for example, one liked timelines, but another preferred text), so their strategy was "to simply chose one". They selected P3's style, and maintained this role assignment for the rest of the course. But, dancers did not include the teacher into their writing negotiations. Because of this, the teacher could not understand some groups' scores: "That's the thing about collaboration, how do I understand what you're writing? Especially if you're writing it in your own style". However, she did not expect technology to 'solve' this, and embraced instead the richness of personal styles. She reflected on this post-study: "Once I had collected all the scores and concatenated them into a global score, it did not matter what personal vocabulary each group used and if I can decipher it at all. We all knew what it referred to in the body, and it was enough to know there was a trace of it in the collective score".

Rather than offering a bridge across a diversity of styles, the insertion of technology sparked conversations on how to notate dance, which led some dancers to find or further define their own styles. Confronted with the task of documenting their work, dancers carefully considered how to write their choreographic phrases, and their reflections were often relative to the available digital and analog technologies. For example, P3 said that Knotation made her reflect on how to notate dance, since "video is not enough" to "detail movement". She mentioned aspects of their composition that could not be captured through video, for example, movement intention or metaphorical images such as "soaking". She added: "We write it 
but I have the impression that with the iPad we could do it in another way. (...) It would require doing something three-dimensional to see how a movement deconstructs little by little, very slowly, because sometimes the video goes too fast. (...) The video also needs to be well shot, but then the fact that we can cut it is great". P5 had a similar view: "So you can take videos... But I think that doesn't use the interesting part of it [Knotation]. (...) I think that drawings allow visualising movement better than video". In some cases, interacting with a feature in Knotation made dancers try to articulate concrete dance concepts. For example, after learning to use floorplans, P2 and P3 said that they were trying to understand what "a trajectory in space" meant for them , and thought about how to better represent its qualities, such as "being irregular" (P2).

\section{DISCUSSION}

\subsection{Studying choreographic collaboration in the wild: Why it matters and why it is messy}

Running this study over the duration of the whole course allowed us to capture not just a number of specific moments and interactions between people and with technology, but also to observe a progression, over time, of their roles, relationships, and needs. This would not have been possible to observe in a laboratory experiment or in any other controlled environment with artificial tasks and roles. For example, we were able to report on how some dancers firstly perceived documenting their dance fragments as 'homework' but later made use of their annotations to revise material, teach each other, negotiate choreographic aspects with the teacher, and how finally seeing the global structure of the piece allowed them to find "meaning in our actions" (P4).

Although the study design and analysis might paint a rather straightforward process in this paper, this did not come without challenges. When reflecting upon this, our perspectives and roles as researchers come as a quite salient aspect. Deploying a tool in a real-world artistic setting added an extra pressure to us as researchers: We felt that the tool had to perform 'beyond properly': fast, reliably, without crashing, without losing any information, without making users lose time, and in general, without generating frustrating moments - and on top of this, expecting it to offer a smooth experience at the height of the artistic process. We were, above all, concerned with proposing a tool that would not fail our study participants, and that would not make them feel observed precisely when they were trying to be creative. The first author is aware that her presence in the studio affected the setting observed, especially at the beginning of the first class, when she set up the camera on a tripod in the corner of the room: "Then they forgot about me until the end of each class. It made me reflect on why we didn't design the study by simply having the teacher set up a camera and then I would analyse video. Was it because I was able to walk around from time to time and overhear conversations? Or take more detailed notes when participants reached for the iPads? Or because I was scared that there would be a bug with Knotation? Probably all of them" (first author, post-study reflection). The second author is also aware of the delicate position she put herself in. She was at the same time one of the researchers investigating the technology's use, the teacher in need of transmitting choreographic knowledge to the students, and the choreographer putting together a student show to be performed in front of an audience. When reflecting on the findings post-study, she stated: "I felt that I was portrayed by the results as a massive dictator, I jokingly asked to hide my identity. But then I thought, this is real life, these tensions, where we go from: horizontality, everyone contributes... to: $O K$, I am in charge here and I need to ensure there is a piece that holds. Knotation supported us, emphasised these relationships and made visible some of the human tensions that occur naturally in these contexts".

We were also concerned about dancers thinking they had to like or even use the tool to please us. This has been discussed, e.g., by Brown et al. [10], who called this particular challenge demand characteristics, where participants adjust their behaviour to match what they perceive to be the researchers' expectations. We did not recognise blatant examples of such modification. However, this might have been influenced by the study being run within a course on dance and technology, which participants were taking for the first time, without their own established practices for documenting dance created collaboratively.

We were, later on, confronted with the always difficult task of writing academically about a fully-fledged research prototype without focusing on its successes, on usability details, and on users' compliments. Fundamentally, we could simply conclude that the tool worked in this real-world setting, but this is not at all the most compelling aspect to highlight and it is, to some extent, expected. We also did not find any concrete story of failure to reflect on, which could have also contributed to the landscape, as argued by Gaver et al. [29] regarding the importance of reporting failed deployments, in order to better distinguish between successful and unsuccessful examples of design, and learn from past mistakes. Instead, rather than an evaluation, we see this field study as a way of uncovering how collaboration in dance making unfolds when mediated by a particular CST. In this paper, we chose to first disclose our initial assumptions - which were later nuanced or contradicted- and then compare them with what we actually observed in the wild. This highlights the value and the need of running field studies to defy researchers' assumptions and inform the design of new tools and in turn, of new studies. We have no doubt that dropping the technology in a different setting would uncover a different, rich set of results - and this is the strength of studying tools in use and not just analysing them in terms of design principles and guidelines.

We align with the conclusions that Brown et al. [10] drew from the difficulty and messiness that comes from studying practices in the wild, and with the risk of engaging with the real world. We see great richness in the mixed positionalities of the authors as investigators and participants. We also highlight the insights gained from participants' reflections on their own practice, sparked by the long-term questioning and experimentation. In line with Brown et al., our methodology reflects the reality of the time spent in the studio with real people, with the complexities of their relationships and of their take on technology.

\subsection{Focusing on what technology did not mediate: Why this is not a problem to solve with CSTs}

A salient aspect of our findings is that the technology did not mediate certain differences among collaborators, and especially their 
power differential. Power dynamics dominated the narrative: The teacher decided who did what, when the technology was to be integrated into the process, when the dancers were to stop using it, and so on. The introduction of Knotation did not fundamentally change the nature of the collaboration that unfolded over time. Of course, collaboration patterns emerged and evolved during the course, as well as participants' roles and their relationship to the technology and to each other, but the pre-existing social construct was preserved and it had a considerable effect in the use of the tool. Such power dynamics are not unique to integrating technologies in dance education. They have been problematised more broadly by researchers addressing the relationships between dance educators and students and even between researchers and participants in the context of action research in dance education [31, 52]. Based on what we were able to observe, the hierarchical difference in the course was not harmful; and although it brought tensions during the generation of movement material, it was accepted by the teacher as a normal part of the process of teaching dance and of collaborating with dancers who are not experienced choreographers themselves. We designed the study so that the first author would avoid actively intervening in the creative process, and would constrain her interaction with dancers to the interviews at scheduled time points, in which she probed into the topics that they decided to highlight. Because of this, the first author did not explicitly ask the dancers to reflect about the power differentials, whereas she had access to the teacher's views even post-study. We recognise this as a limitation of the study, and as an interesting question for future work.

Similarly, Knotation did not mediate the diversity of annotation practices. Two particular stories in our data caught our attention: When the teacher could not understand the dancers' annotations in Knotation; and when P2 said she needed P1, her duo partner, to be able to fully understand P1's documentation of the dance fragment. This made us reflect on whether technology should bridge the gap between personal notating styles. We stand by our decision of designing Knotation to support this diversity, and we argue that CSTs should not try to uniform notating styles. On the one hand, because any attempt to standardise notation practices would necessarily go against the richness and uniqueness of each artist. On the other hand, because there is an inherent value in humanto-human interaction in the context of collaboration: Part of dance making is learning to work with collaborators. Knotation, in fact, while explicitly designed to let participants keep their own style, it also sparked conversations on how to notate dance, which invited them to reflect on the limits of existing representations of dance and digital methods to capture it, such as video.

Crucially, we advocate for designers of CSTs to avoid posing their tools as "solutions" to hierarchical social dynamics in dance making. We argue that attempting to design tools in this way would necessary result in shifting power from people to technology. We now know of many examples illustrating the dangers of imbuing technology with agency over human lives. Such technosolutionism, or what can be defined as the belief that every aspect of life can be addressed with the right technological system, is what Meredith Boussard warned against in her book Artificial Unintelligence How Computers Misunderstand the World [9]. She took as examples driverless cars, AI for standardised student tests, as well as attempts to repair the U.S. campaign finance system. Feminist literature also warns us against implicit gendered differences in computational culture and in the design itself of technology. According to Wajcman in her book Feminist confront technologies [55], technological power is shaped by social distribution of power, and that highly affects the gendered division of labour. Resistance towards technosolutionism has also its roots within HCI: Mark Blythe [6] argued for alternative scenarios (informed debate being one) rather than the traditional problem-solving of the workspace, and Irani [34] showed how Design Thinking, as a problem-solving method that allows to engineer innovative digital solutions, emphasises a racialised understanding of labour that defends North American design and maintains a distribution of labour that serves Western capital interests.

What such activist literature emphasises is the need to re-examine the ambitions of technology as enabling and empowering humans with regards to the hidden agendas that lay behind the innovation discourses - because many of these discourses might serve current distributions of power with regards to gender, race and class among others and even comodify people's experiences and struggles and partake into the neoliberal digital economy. So perhaps, refraining from impregnating technology with more power than it implicitly already beholds can be an alternative. And although dance might appear as a benign field where the stakes are low, there is much to humbly learn about when it comes to how hierarchies play out.

\section{FUTURE WORK}

The presented field study explored the relationships between a choreographer and a group of dancers when the creative process is mediated by a CST, but many other configurations are possible in dance making and worthy of investigating. For example, how would the technological mediation play out when several choreographers collaborate to create a piece, given that the authority would not be concentrated in one person? What about other settings in which the difference of hierarchy between choreographers and dancers is not so marked as in the course we observed? For example, the tool could be dropped into the rehearsals of a dance company with professional dancers that may have previous experience with the representation and archival of dance. These are, in turn, new assumptions that should be contrasted with what happens in the wild. We advocate, thus for running more field studies with fully-fledged research prototypes, given that each deployment will uncover a unique, rich set of results.

\section{CONCLUSIONS}

Collaborative creative processes are shaped by the relationships that exist (and emerge) among content creators. The particularities of each creative field pose specific challenges for the design of relevant interactive tools. Choreography is especially complex, as the roles of choreographers and dancers are defined on the basis of each piece. The ephemeral nature of dance, the variety of writing and representations, and the diversity of styles across choreographers and dance companies all contribute to the difficulty of creating appropriate digital tools. Designing grounded CSTs for choreography, thus, requires us to study how dance artists collaborate, paying attention to the perspectives and expectations of each. 
Knotation acted in this study as a tool, not a powerful nor wilful agent giving (or refusing) power to people. Still, it played the variety of roles described in Section 6.1, according to changing users' needs - going beyond exploration and documentation of ideas, into becoming a support for learning and transmission of material, and negotiation of creative decisions. Importantly, our findings revealed how the introduction of the tool in the creative process served as a pedagogical resource in teaching choreographic collaboration, as it sparked conversations among participants on how to actually collaborate, what collaboration entails, how the concepts of trust and agency play out in this context, as well as how to notate dance and transmit the resulting patterns. Knotation, together with the methods included in our study (in particular, the debriefings and interviews), constituted thus an intervention that invited participants to reflect about key aspects of not just choreography per se, but the human and collaborative component involved in creating dance. We conclude that as dance artists continue to (re)define collaboration on the base of each dance piece, designers of CSTs for choreography should take this complexity into consideration, both when creating new tools and when studying them in situ.

\section{ACKNOWLEDGMENTS}

We deeply thank participants for taking part in this study and reviewers for their constructive comments. Marianela is also grateful to Pedro Sanches for his kind feedback on a first draft. This research was partially funded by ERC no. 321135, CREATIV.

\section{REFERENCES}

[1] Sarah Fdili Alaoui, Baptiste Caramiaux, Marcos Serrano, and Frédéric Bevilacqua. 2012. Movement Qualities as Interaction Modality. In Proceedings of the Designing Interactive Systems Conference (Newcastle Upon Tyne, United Kingdom) (DIS '12) Association for Computing Machinery, New York, NY, USA, 761-769. https: //doi.org/10.1145/2317956.2318071

[2] Sarah Fdili Alaoui, Kristin Carlson, and Thecla Schiphorst. 2014. Choreography as Mediated through Compositional Tools for Movement: Constructing A Historical Perspective. In Proceedings of the 2014 International Workshop on Movement and Computing (Paris, France) (MOCO '14). Association for Computing Machinery, New York, NY, USA, 1-6. https://doi.org/10.1145/2617995.2617996

[3] Steve Benford, Chris Greenhalgh, Andy Crabtree, Martin Flintham, Brendan Walker, Joe Marshall, Boriana Koleva, Stefan Rennick Egglestone, Gabriella Giannachi, Matt Adams, et al. 2013. Performance-led research in the wild. ACM Transactions on Computer-Human Interaction (TOCHI) 20, 3 (2013), 1-22.

[4] Johannes Birringer. 2002. Dance and Media Technologies. PA7: A fournal of Performance and Art 24, 1 (2002), 84-93. https://doi.org/10.1162/152028101753401811

[5] Lynne Anne Blom and L. Tarin Chaplin. 1982. The Intimate Act Of Choreography. University of Pittsburgh Press, Pittsburgh.

[6] Mark Blythe. 2017. Research Fiction: Storytelling, Plot and Design. In Proceedings of the 2017 CHI Conference on Human Factors in Computing Systems (Denver, Colorado, USA) (CHI '17). Association for Computing Machinery, New York, NY, USA, 5400-5411. https://doi.org/10.1145/3025453.3026023

[7] Virginia Braun and Victoria Clarke. 2006. Using thematic analysis in psychology Qualitative Research in Psychology 3, 2 (2006), 77-101.

[8] Virginia Braun and Victoria Clarke. 2019. Reflecting on reflexive thematic analysis Qualitative Research in Sport, Exercise and Health 11, 4 (2019), 589-597.

[9] Meredith Broussard. 2018. Artificial unintelligence: How computers misunderstand the world. MIT Press, Cambridge, Massachusetts.

[10] Barry Brown, Stuart Reeves, and Scott Sherwood. 2011. Into the Wild: Challenges and Opportunities for Field Trial Methods. In Proceedings of the SIGCHI Conference on Human Factors in Computing Systems (Vancouver, BC, Canada) (CHI '11). Association for Computing Machinery, New York, NY, USA, 1657-1666. https: //doi.org/10.1145/1978942.1979185

[11] Jo Butterworth. 2004. Teaching choreography in higher education: A process continuum model. Research in dance education 5, 1 (2004), 45-67.

[12] Kristin Carlson, Herbert H. Tsang, Jordon Phillips, Thecla Schiphorst, and Tom Calvert. 2015. Sketching Movement: Designing Creativity Tools for In-situ, Whole-body Authorship. In Proceedings of the 2Nd International Workshop on
Movement and Computing (Vancouver, British Columbia, Canada) (MOCO '15). ACM, New York, NY, USA, 68-75. https://doi.org/10.1145/2790994.2791007

[13] Erin A. Carroll, Danielle Lottridge, Celine Latulipe, Vikash Singh, and Melissa Word. 2012. Bodies in Critique: A Technological Intervention in the Dance Production Process. In Proceedings of the ACM 2012 Conference on Computer Supported Cooperative Work (Seattle, Washington, USA) (CSCW'12). ACM, New York, NY, USA, 705-714. https://doi.org/10.1145/2145204.2145311

[14] Teresa Cerratto-Pargman, Chiara Rossitto, and Louise Barkhuus. 2014. Understanding Audience Participation in an Interactive Theater Performance. In Proceedings of the 8th Nordic Conference on Human-Computer Interaction: Fun, Fast, Foundational (Helsinki, Finland) (NordiCHI '14). Association for Computing Machinery, New York, NY, USA, 608-617. https://doi.org/10.1145/2639189.2641213

[15] Erin Cherry and Celine Latulipe. 2014. Quantifying the Creativity Support of Digital Tools Through the Creativity Support Index. ACM Trans. Comput.-Hum. Interact. 21, 4, Article 21 (June 2014), 25 pages.

[16] Luke Church, Nick Rothwell, Marc Downie, Scott DeLahunta, and Alan F. Blackwell. 2012. Sketching by Programming in the Choreographic Language Agent. In Proceedings of the 24th Annual Workshop of the Psychology of Programming Interest Group, PPIG 2012, London, UK, November 21-23, 2012. Psychology of Programming Interest Group, London, UK, 16. http://ppig.org/library/paper/sketchingprogramming-choreographic-language-agent

[17] Marianela Ciolfi Felice. 2018. Supporting expert creative practice. Ph.D. Dissertation. Université Paris Saclay.

[18] Marianela Ciolfi Felice, Sarah Fdili Alaoui, and Wendy E. Mackay. 2016. How Do Choreographers Craft Dance?: Designing for a Choreographer-Technology Partnership. In Proceedings of the 3rd International Symposium on Movement and Computing (Thessaloniki, GA, Greece) (MOCO '16). ACM, New York, NY, USA, Article 20, 8 pages.

[19] Marianela Ciolfi Felice, Sarah Fdili Alaoui, and Wendy E. Mackay. 2018. Knotation: Exploring and Documenting Choreographic Processes. In Proceedings of the 2018 CHI Conference on Human Factors in Computing Systems (Montreal QC, Canada) (CHI '18). Association for Computing Machinery, New York, NY, USA, 1-12. https://doi.org/10.1145/3173574.3174022

[20] Peter Dalsgaard. 2018. Rethinking Interaction in Creative Work.

[21] Scott deLahunta and Philip Barnard. 2005. What's in a Phrase. Tanz im KopfL farbuch 15 der Gesellschaft für Tanzforschung -, - (2005), 253-266.

[22] Scott deLahunta, Wayne McGregor, and Alan Blackwell. 2004. Transactables. Performance Research 9, 2 (2004), 67-72.

[23] Scott deLahunta and Norah Zuniga Shaw. 2006. Constructing memories: Creation of the choreographic resource. Performance Research 11, 4 (2006), 53-62.

[24] Steven Dow, Manish Mehta, Ellie Harmon, Blair MacIntyre, and Michael Mateas. 2007. Presence and Engagement in an Interactive Drama. In Proceedings of the SIGCHI Conference on Human Factors in Computing Systems (San Jose, California, USA) (CHI '07). Association for Computing Machinery, New York, NY, USA, 1475-1484. https://doi.org/10.1145/1240624.1240847

[25] Katerina El Raheb, Aristotelis Kasomoulis, Akrivi Katifori, Marianna Rezkalla, and Yannis Ioannidis. 2018. A Web-Based System for Annotation of Dance Multimodal Recordings by Dance Practitioners and Experts. In Proceedings of the 5th International Conference on Movement and Computing (Genoa, Italy) (MOCO '18). Association for Computing Machinery, New York, NY, USA, Article 8, 8 pages. https://doi.org/10.1145/3212721.3212722

[26] Sarah Fdili Alaoui. 2019. Making an Interactive Dance Piece: Tensions in Integrating Technology in Art. In Proceedings of the 2019 on Designing Interactive Systems Conference (San Diego, CA, USA) (DIS '19). Association for Computing Machinery, New York, NY, USA, 1195-1208. https://doi.org/10.1145/3322276.3322289

[27] Sarah Fdili Alaoui, Frédéric Bevilacqua, Bertha Bermudez Pascual, and Christian Jacquemin. 2013. Dance interaction with physical model visuals based on movement qualities. International Journal of Arts and Technology 6, 4 (2013), 357-387.

[28] Sarah Fdili Alaoui, Thecla Schiphorst, Shannon Cuykendall, Kristin Carlson, Karen Studd, and Karen Bradley. 2015. Strategies for Embodied Design: The Value and Challenges of Observing Movement. In Proceedings of the 2015 ACM SIGCHI Conference on Creativity and Cognition (Glasgow, United Kingdom) (C\&\#38;C '15). ACM, New York, NY, USA, 121-130. https://doi.org/10.1145/2757226.2757238

[29] William Gaver, John Bowers, Tobie Kerridge, Andy Boucher, and Nadine Jarvis. 2009. Anatomy of a Failure: How We Knew When Our Design Went Wrong, and What We Learned from It. In Proceedings of the SIGCHI Conference on Human Factors in Computing Systems (Boston, MA, USA) (CHI '09). Association for Computing Machinery, New York, NY, USA, 2213-2222. https://doi.org/10.1145/ 1518701.1519040

[30] Sara Gibbons. 2015. Co-Authorship in Action: Curation \& Collaboration in American post-Judson Dance. Honor theses -, - (2015), Paper 771.

[31] Miriam Giguere. 2015. Dance education action research: a twin study. Research in Dance Education 16, 1 (2015), 16-32. https://doi.org/10.1080/14647893.2014.971231 arXiv:https://doi.org/10.1080/14647893.2014.971231

[32] Jonathan Hook, John McCarthy, Peter Wright, and Patrick Olivier. 2013. Waves: Exploring Idiographic Design for Live Performance. In Proceedings of the SIGCHI Conference on Human Factors in Computing Systems (Paris, France) (CHI '13). 
Association for Computing Machinery, New York, NY, USA, 2969-2978. https: //doi.org/10.1145/2470654.2481412

[33] Stacy Hsueh, Sarah Fdili Alaoui, and Wendy E. Mackay. 2019. Deconstructing Creativity: Non-Linear Processes and Fluid Roles in Contemporary Music and Dance. Proc. ACM Hum.-Comput. Interact. 3, CSCW, Article 203 (Nov. 2019), 21 pages. https://doi.org/10.1145/3359305

[34] Lilly Irani. 2018. "Design thinking": Defending Silicon Valley at the apex of global labor hierarchies. Catalyst: Feminism, Theory, Technoscience 4, 1 (2018), - .

[35] David Kirsh, Dafne Muntanyola, R Joanne Jao, Amy Lew, and Matt Sugihara. 2009. Choreographic methods for creating novel, high quality dance. In Proceedings, DESFORM 5th international workshop on Design \& Semantics \& Form. -, -, 188-195.

[36] Michael Klien. 2007. Choreography: A pattern language. Kybernetes 36, 7/8 (2007), 1081-1088

[37] Celine Latulipe. 2013. The Value of Research in Creativity and the Arts. In Proceedings of the 9th ACM Conference on Creativity \& Cognition (Sydney, Australia) (C\&C '13). ACM, New York, NY, USA, 1-10. https://doi.org/10.1145/2466627.2485921

[38] Celine Latulipe, David Wilson, Sybil Huskey, Berto Gonzalez, and Melissa Word. 2011. Temporal Integration of Interactive Technology in Dance: Creative Process Impacts. In Proceedings of the 8th ACM Conference on Creativity and Cognition (Atlanta, Georgia, USA) (C\&\#38;C '11). ACM, New York, NY, USA, 107-116. https: //doi.org/10.1145/2069618.2069639

[39] Lian Loke, Dagmar Reinhardt, and Jodie McNeilly. 2015. Performer-Machine Scores for Choreographing Bodies, Interaction and Kinetic Materials. In Proceedings of the 2nd International Workshop on Movement and Computing (Vancouver, British Columbia, Canada) (MOCO '15). Association for Computing Machinery, New York, NY, USA, 52-59. https://doi.org/10.1145/2790994.2790999

[40] Lian Loke and Toni Robertson. 2010. Studies of dancers: Moving from experience to interaction design. International fournal of Design 4, 2 (2010), -.

[41] Wendy E. Mackay. 2002. Using video to support interaction design. DVD Tutorial, CHI 2 (2002), 5

[42] Raul Masu, Nuno N. Correia, Stephan Jurgens, Jochen Feitsch, and Teresa Romão 2020. Designing Interactive Sonic Artefacts for Dance Performance: An Ecological Approach. In Proceedings of the 15th International Conference on Audio Mostly (Graz, Austria) (AM '20). Association for Computing Machinery, New York, NY, USA, 122-129. https://doi.org/10.1145/3411109.3412297

[43] Claudia Ribeiro, Rafael Kuffner, and Carla Fernandes. 2018. Virtual Reality Annotator: A Tool to Annotate Dancers in a Virtual Environment. In Digital Cultural Heritage. Springer, -, 257-266.

[44] Jean-Philippe Rivière, Sarah Fdili Alaoui, Baptiste Caramiaux, and Wendy E. Mackay. 2019. Capturing Movement Decomposition to Support Learning and Teaching in Contemporary Dance. Proc. ACM Hum.-Comput. Interact. 3, CSCW, Article 86 (Nov. 2019), 22 pages. https://doi.org/10.1145/3359188

[45] Jean-Philippe Rivière, Sarah Fdili Alaoui, Baptiste Caramiaux, and Wendy E. Mackay. 2021. Exploring the Role of Artifacts in Collective Dance Re-Staging. Proc. ACM Hum.-Comput. Interact. 5, CSCW1, Article 108 (April 2021), 22 pages. https://doi.org/10.1145/3449182

[46] Yvonne Rogers and Paul Marshall. 2017. Research in the Wild. Synthesis Lectures on Human-Centered Informatics 10, 3 (2017), i-97.

[47] Bonnie Rowell. 2000. United Kingdom: An expanding map. In Europe dancing. Perspectives on theatre dance and cultural identity, A. Grau and S. Jordan (Eds.) London: Routledge, London, 188-212.

[48] Thecla Schiphorst, Tom Calvert, Catherine Lee, Christopher Welman, and Severin Gaudet. 1990. Tools for interaction with the creative process of composition. In Proceedings of the SIGCHI Conference on Human Factors in Computing Systems. ACM, New York, 167-174.

[49] Ben Shneiderman. 2007. Creativity support tools: Accelerating discovery and innovation. Commun. ACM 50, 12 (2007), 20-32.

[50] Vikash Singh, Celine Latulipe, Erin Carroll, and Danielle Lottridge. 2011. The choreographer's notebook: a video annotation system for dancers and choreographers. In Proceedings of the 8th ACM conference on Creativity and Cognition. ACM, New York, 197-206.

[51] Susan Leigh Star. 1989. The structure of ill-structured solutions: heterogeneous problem-solving, boundary objects and distributed artificial intelligence. Distributed Artificial Intelligence 2 (1989), 37-54.

[52] Susan W Stinson. 1998. Seeking a feminist pedagogy for children's dance. Dance, power, and difference: Critical and feminist perspectives on dance education -, (1998), 23-47.

[53] Robyn Taylor, Guy Schofield, John Shearer, Jayne Wallace, Peter Wright, Pierre Boulanger, and Patrick Olivier. 2011. Designing from within: Humanaquarium. In Proceedings of the SIGCHI Conference on Human Factors in Computing Systems (Vancouver, BC, Canada) (CHI '11). Association for Computing Machinery, New York, NY, USA, 1855-1864. https://doi.org/10.1145/1978942.1979211

[54] Rudolf von Laban and Lisa Ullmann. 1948. Modern educational dance. Macdonald \& Evans, -.

[55] Judy Wajcman. 1991. Feminism confronts technology. Penn State Press, Pennsylvania.

[56] Zhenyu Yang, Bin Yu, Wanmin Wu, Ross Diankov, and Ruzena Bajscy. 2006 Collaborative Dancing in Tele-Immersive Environment. In Proceedings of the 14th ACM International Conference on Multimedia (Santa Barbara, CA, USA) (MM '06). Association for Computing Machinery, New York, NY, USA, 723-726. https://doi.org/10.1145/1180639.1180793 